\title{
Six simple questions contra the delay
}

\author{
Felix C. Ringshausen ${ }^{1}$, Tobias Welte ${ }^{1}$ and Albert Nienhaus ${ }^{2}$
}

Affiliations: 'Dept of Respiratory Medicine, Hannover Medical School, Hannover, and ${ }^{2}$ Institute for Health Services Research in Dermatology and Nursing, University Medical Center Hamburg-Eppendorf, Hamburg, Germany.

Correspondence: F.C. Ringshausen, Dept of Respiratory Medicine, Hannover Medical School, Carl-NeubergStrasse 1, 30625 Hannover, Germany. E-mail: Ringshausen.Felixamh-hannover.de

0 @ERSpublications

Six simple questions could quickly identify active TB in patients with community-acquired pneumonia admitted to hospital http://ow.ly/r3cqt

During the past decades, the incidence of tuberculosis (TB) has steadily declined in most west European countries, and many physicians will no longer encounter TB in their patients. Along with the declining practical experience of physicians, the chance for delay in diagnosing TB increases. An delayed diagnosis of TB index cases is the most important risk factor for the nosocomial transmission of Mycobacterium tuberculosis (MTB) [1], as numerous unprotected contacts among healthcare workers and inpatients may result before the diagnosis of TB is established and respiratory isolation is applied [2,3]. The clean-up after such a mishap can be complicated and time-consuming, as the individuals concerned need to be identified and contacted. Patients might have been already discharged, or healthcare workers may not be aware of exposure to a TB index case for days or even weeks [4]. However, the difficulty is not over, even when the respective contacts have been identified. As there is no perfect test for the diagnosis of TB infection, the question arises of which test should be used and who should be tested with this assay in order to obtain optimal results. Only close contacts should be tested in order to improve the positive predictive value of the contact screening regardless of the test used. But what is a close contact in a hospital setting? Cumulative exposure to a smear-positive index case for $8 \mathrm{~h}$ or more is proposed as an orientation. However, this is merely an inspired guess. In comparison to the tuberculin skin test, the emergence of interferon- $\gamma$ release assays (IGRAs) has facilitated screening after unprotected TB exposure, as there is no booster phenomenon after repeat testing, no need for a second appointment for test reading and no influence of bacillus Calmette-Guérin vaccination on subsequent test results. However, even with IGRAs, many questions remain unanswered. IGRAs are not able to distinguish remote from recent infection and their ability to predict progression to active disease appears to be limited so far. While progression rates of up to $14 \%$ have been reported for close contacts with a positive IGRA result $[5,6]$, two recent systematic reviews and metaanalyses indicate considerably lower progression risks $[7,8]$. Therefore, even after a positive IGRA is observed, the question remains regarding what to do next. Of course, active TB needs to be excluded by chest radiograph. But should we propose preventive chemotherapy to all contacts with a positive IGRA result, well knowing that most of these subjects will never develop active TB anyway?

When a patient with community-acquired pneumonia (CAP) is admitted to the hospital, MTB could almost always be the causative pathogen. However, administering an IGRA for all CAP patients would be a waste of time and money, as current IGRAs are not able to distinguish active TB from latent TB infection. Moreover, the sensitivity of IGRAs may be compromised by the immunological status of the patient. Thus, a negative IGRA does not rule out active TB. Furthermore, active TB might be suspected in far too many contacts in populations with a high prevalence of (remote) latent $\mathrm{TB}$ infection and precious time may be lost while sputum results are awaited.

Received: Nov 192013 | Accepted: Nov 202013

Conflict of interest: Disclosures can be found alongside the online version of this article at www.erj.ersjournals.com

Copyright @ERS 2014 
There is a simple and very basic approach to solving this dilemma: asking the right questions! As presented in the large cohort study by CAVALLAZZ et al. [9] in this issue of the European Respiratory Journal, there are six simple, though crucial, questions for suspecting active TB in patients admitted to the hospital with CAP. These six questions were derived from a set of 22 questions which are known to provide evidence for the diagnosis of TB [10]. However, while 22 questions are hard to remember and take longer to pose, these few simple questions are, besides being more accurate according to the present study [9], considerably easier to remember; as a result, they can even be applied as a bedside tool for predicting the risk of TB in hospitalised patients with CAP in the setting of a busy emergency department with all its time constraints and distractors. Unsurprisingly, weight loss, night sweats, haemoptysis, exposure to MTB and younger age ( $<65$ years) are among these questions. As a result, compared to the original Centers of Disease Control and Prevention score, a significantly improved TB risk assessment was achieved with a subset of risk factors, along with the presence of upper lobe infiltrates.

As respiratory isolation is an effective intervention to prevent the nosocomial transmission of TB and to contribute to TB control [11], the Community-Acquired Pneumonia Organization (CAPO) TB risk score has the potential to contribute to optimal patient care and to facilitate the reasonable allocation of healthcare resources by simplifying and improving TB risk assessment of hospitalised patients with CAP. In this regard, the implementation of the CAPO TB risk score may reduce the delay of identifying potential TB index cases in order to prevent nosocomial transmission to healthcare workers and other patients. Moreover, it may improve the prognosis of TB patients due to timely initiation of adequate treatment [12] and, in addition, guide the choice of empirical antibiotic treatment with regard to the yield of diagnostic tests for MTB [13]. However, it should be noted that atypical clinical and radiological presentations frequently occur among elderly and immunocompromised patients [14].

Future approaches to further improve the informative value of the CAPO TB risk score could encompass the inclusion of the duration of symptoms [15] and the addition of a biomarker different from the MTBspecific interferon $\gamma$ response, e.g. C-reactive protein or procalcitonin, which are expected to be low in subjects with CAP due to MTB [16].

As mentioned by the authors, their results need prospective validation in different well-defined clinical settings, particularly given the heterogeneous epidemiology of TB among the 33 contributing countries across Africa, Asia, Australia, Europe, Latin America and USA/Canada, where, in addition, empirical treatment strategies of CAP may differ significantly according to the prevalence of penicillin-resistant pneumococci, the predominant pathogen in CAP, and other multidrug-resistant pathogens [17].

\section{References}

1 de Vries G, Sebek MM, Lambregts-van Weezenbeek CS. Healthcare workers with tuberculosis infected during work. Eur Respir J 2006; 28: 1216-1221.

2 Greenaway C, Menzies D, Fanning A, et al. Delay in diagnosis among hospitalized patients with active tuberculosispredictors and outcomes. Am J Respir Crit Care Med 2002; 165: 927-933.

Nienhaus A, Schablon A, Ringshausen FC, et al. TB as an occupational disease. Eur Respir Monogr 2012; 58: 219-229.

4 Ringshausen FC, Schlosser S, Nienhaus A, et al. In-hospital contact investigation among health care workers after exposure to smear-negative tuberculosis. J Occup Med Toxicol 2009; 4: 11.

5 Diel R, Loddenkemper R, Niemann S, et al. Negative and positive predictive value of a whole-blood interferon- $\gamma$ release assay for developing active tuberculosis: an update. Am J Respir Crit Care Med 2011; 183: 88-95.

6 Haldar P, Thuraisingam H, Patel H, et al. Single-step QuantiFERON screening of adult contacts: a prospective cohort study of tuberculosis risk. Thorax 2013; 68: 240-246.

7 Diel R, Loddenkemper R, Nienhaus A. Predictive value of interferon-gamma release assays and tuberculin skin testing for progression from latent TB infection to disease state: a meta-analysis. Chest 2012; 142: 63-75.

8 Rangaka MX, Wilkinson KA, Glynn JR, et al. Predictive value of interferon-gamma release assays for incident active tuberculosis: a systematic review and meta-analysis. Lancet Infect Dis 2012; 12: 45-55.

9 Cavallazzi R, Wiemken T, Christensen D, et al. Predicting Mycobacterium tuberculosis in patients with communityacquired pneumonia. Eur Respir J 2014; 43: 178-184.

10 Centers for Disease Control and Prevention. Guidelines for preventing the transmission of Mycobacterium tuberculosis in health-care settings, 2005. MMWR Recomm Rep 2005; 54: 1-141.

11 Raviglione M, Marais B, Floyd K, et al. Scaling up interventions to achieve global tuberculosis control: progress and new developments. Lancet 2012; 379: 1902-1913.

12 Sreeramareddy CT, Panduru KV, Menten J, et al. Time delays in diagnosis of pulmonary tuberculosis: a systematic review of literature. BMC Infect Dis 2009; 9: 91.

13 Dooley KE, Golub J, Goes FS, et al. Empiric treatment of community-acquired pneumonia with fluoroquinolones, and delays in the treatment of tuberculosis. Clin Infect Dis 2002; 34: 1607-1612.

14 Geng E, Kreiswirth B, Burzynski J, et al. Clinical and radiographic correlates of primary and reactivation tuberculosis: a molecular epidemiology study. JAMA 2005; 293: 2740-2745.

15 Liam CK, Pang YK, Poosparajah S. Pulmonary tuberculosis presenting as community-acquired pneumonia. Respirology 2006; 11: 786-792.

16 Ugajin M, Miwa S, Shirai M, et al. Usefulness of serum procalcitonin levels in pulmonary tuberculosis. Eur Respir J 2011; 37: 371-375.

17 Welte T, Kohnlein T. Global and local epidemiology of community-acquired pneumonia: the experience of the CAPNETZ Network. Semin Respir Crit Care Med 2009; 30: 127-135. 